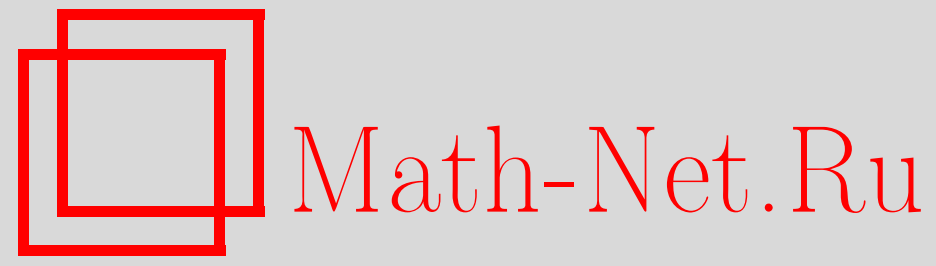

Общероссийский математический портал

В. А. Файзиев, Псевдохарактеры на правильных произведениях групп, Матем. заметки, 1996, том 59, выпуск 2, 314-316

DOI: https://doi.org/10.4213/mzm1719

Использование Общероссийского математического портала MathNet.Ru подразумевает, что вы прочитали и согласны с пользовательским соглашением http://www.mathnet.ru/rus/agreement

Параметры загрузки:

IP : 35.173 .219 .12 
26 апреля 2023 г., 11:23:02

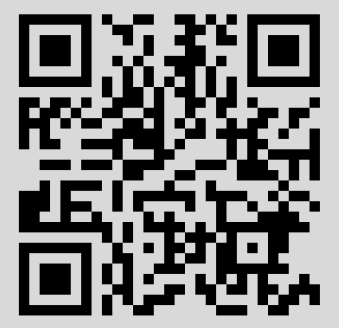




\section{ПСЕВДОХАРАКТЕРЫ НА ПРАВИЛЬНЫХ ПРОИЗВЕДЕНИЯХ ГРУПП}

\section{В. А. Файзиев}

Напомним (см. [1]), что псевдохарактером группы $G$ называется вещественнозначная функция $f$ на $G$, удовлетворяющая следующим условиям: 1) $f\left(x^{n}\right)=n f(x)$ $\forall n \in \mathbb{Z}, \forall x \in G ; 2)$ множество $\{f(x y)-f(x)-f(y) \mid x, y \in G\}$ ограничено. Множество всех псевдохарактеров группы $S$ обозначим через $P X(S)$. Очевидно, что $P X(S)$ является вещественным линейным пространством относительно обычных операций. Его подпространство, состоящее из вещественных аддитивных характеров, обозначим через $X(S)$. Пусть $\tau$ эпиморфиизм группы $G$ на группу $H$ и $\varphi \in P X(H)$, тогда ясно, что функция $\varphi \circ \tau$ будет псевдохарактером группы $G$, а отображение $\tau^{*}: \varphi \rightarrow \varphi$ ○ является вложением пространства $P X(H)$ в $P X(G)$.

Пусть $G=\prod_{i \in J} A_{i}$ - правильное произведение групп $A_{i}, i \in J$, (см. [2], [3]), тогда для любого $i \in J$ отображение $\tau_{i}$ такое, что $\tau_{i}\left(a_{i}\right)=a_{i} \forall a_{i} \in A_{i}$ и $\tau_{i}\left(a_{j}\right)=1$ $\forall a_{j} \in A_{j}, i \neq j$, продолжается до эпиморфизма группы $G$ на $A_{i}$. Эти эпиморфизмы $\tau_{i}$ назовем естественными эпиморфизмами группы $G$ на сомножители, они индуцируют естественные вложения $\tau_{i}^{*}: P X\left(A_{i}\right) \rightarrow P X(G)$. В дальнейшем будем считать, что пространства $P X\left(A_{i}\right)$ вложены в $P X(G)$ естественными вложениями $\tau_{i}^{*}$. Декартовой подгруппой правильного произведения $G=\prod_{i \in J} A_{i}$ будем назьвать нормальный делитель $D$ группы $G$, порожденный множеством коммутаторов $\left\{\left[a_{i}, a_{j}\right] \mid a_{i} \in A_{i}, a_{j} \in A_{j}, i \neq j, i, j \in J\right\}$. Декартова подгруппа группы $G=\prod_{i \in J} A_{i}$ является ядром естественного эпиморфизма группы $\prod_{i \in J} A_{i}$ на прямое произведение $G=\bigotimes_{i \in J} A_{i}$. Если правильное произведение групп $A_{i}, i \in J$ вырождается в их свободное произведение, то декартова подгруппа является свободной группой. Грюнбергом в [4] указана система свободных образующих этой группы, которая состоит из правосторонних коммутаторов $\left[a_{i_{1}}, a_{i_{2}}, \ldots, a_{i_{k}}\right]$, где все $i_{m}$ различны, $i_{1}>i_{2}, i_{2}<i_{3}<\cdots<i_{k}, k \geqslant 2, a_{i_{m}} \in A_{i_{m}}, a_{i_{m}} \neq 1$, $m=1,2, \ldots, k$ (предполагается, что зафиксирована некоторая линейная упорядоченность множества индексов $J$ ). Ясно, что образ этой системы образующих при естественном эпиморфизме свободного произведения $G=\bigotimes_{i \in J} A_{i}$ на правильное $G=\prod_{i \in J} A_{i}$ будет системой образующих, которую будем называть грюнберговой, декартовой подгруппы в $G=\prod_{i \in J} A_{i}$. 
Пусть $G=\prod_{i \in J} A_{i}$ правильное произведение групп $A_{i}, i \in J$, и $D$ - его декартова подгруппа. Будем говорить, что псевдохарактер $\varphi$ группы $D$ инвариантен относительно действия группы $G$, если для любого $x$ из $D$ и любого $g$ из $G$ вьполняется равенство $\varphi\left(x^{g}\right)=\varphi\left(g^{-1} x g\right)=\varphi(x)$.

Обозначим через $B P X(D, G)$ подпространство $P X(D)$, состоящее из псевдохарактеров группы $D$, инвариантных относитељно действия группы $G$, ограниченных на грюнберговой системе образующих и на множестве

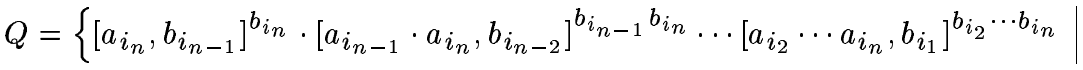

$$
\begin{aligned}
& \left.i_{1}<i_{2}<\cdots<i_{n}, n \geqslant 2 ; a_{i_{m}}, b_{i_{m}} \in A_{i_{m}}, i_{m} \in J\right\} \text {. }
\end{aligned}
$$

Лемма 1. Пусть $A=\prod_{i \in J} A_{i}$ - прямое произведение групп $A_{i}, i \in J$. Тогда всякий псевдохарактер группы А разлагается и единственным образом в $\ell_{1}-$ сумму псевдохарактеров сомножителей. Это означает, что если $\varphi \in P X(A), \varphi_{i}=\left.\varphi\right|_{A_{i}} u$

$$
\begin{aligned}
\Delta & =\sup \{\varphi(x y)-\varphi(x)-\varphi(y) \mid x, y \in A\}, \\
\Delta_{i} & =\sup \left\{\varphi_{i}(x y)-\varphi_{i}(x)-\varphi_{i}(y) \mid x, y \in A_{i}\right\},
\end{aligned}
$$

то существует не более чем счетное подмножество $J_{0} \in J$ такое, что $\Delta_{i} \neq 0$ в том и только в том случае, когда $i \in J_{0} u$

$$
\Delta=\sum_{i \in J_{0}} \Delta_{i}, \quad \varphi=\sum_{i \in J} \varphi_{i} .
$$

В силу естественного эпиморфизма группы $G=\prod_{i \in J} A_{i}$ на группу $A=\bigotimes_{i \in J} A_{i}$ пространство $P X(A)$ вкладывается в $P X(G)$.

Теорема 1. Пусть $G=\prod_{i \in J} A_{i}-$ правильное произведение групп и декартова подгруппа в $\prod_{i \in J} A_{i}$. Тогда существует такое вложение $B P X(D, G)$ в $P X(G)$, что $P X(G)=B P X(D, G)+P X\left(\bigotimes_{i \in J} A_{i}\right)$

Частным случаем правильных произведений групп являются вербальные произведения, введенные в [5].

СлЕДСтвиЕ 1. Пусть $k$ - натуральное число $u$ о означает $k$-е нильпотентное, $k$-е разрешимое или $k$-е бернсайдово произведение групп. Тогда для любого набора групп $A_{i}, i \in J$, справедливо равенство $P X\left(\prod_{i \in J} A_{i}\right)$ $=P X\left(\otimes_{i \in J} A_{i}\right)$.

Пусть $G=A$ wr $B$ - сплетение групп $A$ и $B, K=\bigotimes_{b \in B} A(b)(A(b) \cong A)$ - его базовая группа. Каждому элементу $\varphi$ из $P X(A)$ сопоставим вещественную функцию $\bar{\varphi}$ на $K$ по правилу

$$
\bar{\varphi}\left(a_{1}\left(b_{1}\right) \cdot a_{2}\left(b_{2}\right) \cdots a_{n}\left(b_{n}\right)\right)=\sum_{i=1}^{n} \varphi\left(a_{i}\right) .
$$

Тогда отображение $\pi: \varphi \rightarrow \bar{\varphi}$ будет вложением $X(A)$ в $X(K, B)$, а если группа $B$ конечная, то $\pi$ будет вложением $P X(A)$ в $P X(K, B)$. 
ПРЕДЛОЖЕНИЕ 1. Пусть $G=A$ wr $B$. Тогдa

1) если группа $B$ бесконечная, то $P X(G)=P X(B)+X(A)$;

2) если В конечная, то $P X(G)=P X(A)$.

Теорема 2. Пусть $H=A \mathrm{wr}_{v} B-v$-вербальное сплетение групп $A u B$, $\prod_{b \in B}^{v} A(b)$ - его базовая группа, а $D$ - декартова подгруппа в $\prod_{b \in B}^{v} A(b)$. Tогдa

$$
P X(H)=P X(A \text { wr } B) \dot{+} B P X(D, H),
$$

где $\operatorname{BPX}(D, H)$ означает пространство псевдохарактеров группь $D$, инвариантных относительно действия группы $H$, ограниченных на мнохестве $Q$ и на грюнберговой системе образующих декартовой подгруппы $D$.

СледствиЕ 2. Если $k \in \mathbb{N} u H=A \mathrm{wr}_{v} B-k$-e нильпотентное, $k-e$ разрешимое или $k$-е бернсайдово сплетение, то $P X(H)=P X(A$ wr $B)$.

Математический институт

\section{СПИСОК ЦИТИРОВАННОЙ ЛИТЕРАТУРЫ}

1. Штерн А. И. // Функцион. анализ и его прилож. 1991. Т. 25. № 2. С. 70-73. 2. Головин О. Н. // Матем. сб. 1950. Т. 27. С. 427-454. 3. Курош А. Г. Теория групп. М.: Наука, 1967. 4. Gruenberg K. // Proc. London Math. Soc. 1957. V. 7. № 25. P. 29-62 . 5. Moran S. // Proc. London Math. Soc. 1956. V. 6. № 24. P. 581-596. 